\title{
An Intelligent Drawer System
}

\author{
Dong Xie, Shilin Li, Qinqin Xiao, Shengxiong Wei, Yaxi Li, Jian Tong \\ Information School, Hunan University of Humanities, Science and Technology \\ Loudi, China
}

\begin{abstract}
This design adopts single chip microcontroller (STM32F103) as the minimum system. The independent keystroke and single chip are the control module of the fingerprint. Fingerprint identification module and WIFI module constitute information acquisition module. The dc motor and the lock - clasp physical components constitute the electric locking module. Three LED lights constitute a status indicator module. The system is powered by a battery. The system has the advantages of simple structure, low production cost, high control accuracy and strong anti-interference ability.
\end{abstract}

Keywords: Intelligent drawer, Fingerprint identification, STM32F103.

\section{Introduction}

New times give people new pursuits. People in the past sought cheap and durable, and now people are more apt to be smart and practical. Many designers like to re-create existing objects [1]. This allows for more powerful and practical items. We also hope to create a more attractive and more human smart drawer by creating the innovation in the drawer [2].

\section{Selection and Demonstration of the System Scheme}

The main control center of the system is divided into two categories:

Scheme 1: the system adopts 51 series single-chip microcomputer. Single chip microcomputer is a more commonly used 8 - bit microcontroller. The advantages of the McS-51 system include the typical structure and the centralized management of the perfect bus special registers, numerous logical bit operation functions and the rich instruction system oriented to control [3]. It also has advantages including simple development, cheap price, and good generic type and so on. And it has a single-chip kernel based on complex instructions (CISC).But 51 microphones didn't react fast enough. It takes 12 oscillations to execute a single cycle command [4]. So it's more suitable for devices that control simple bits of information. Because it is slow, $51 \mathrm{MCU}$ is not the best choice for a high speed area. In addition, 51 MCU USES a lot of power.

Scheme 2: the system ado rich set of timers and I/O ports. And it has low power consumption, stable performance, reliable and other advantages. It has up to $64 \mathrm{~K}$ bytes of built-in SRAM, so it can achieve multifunctional control of the system. It can also carry out high-speed data acquisition and processing. This series of single chip is exactly in line with our requirements.

The system theory scheme architecture is shown in Figure 1. The stereo rectangle is the box body of the whole experiment. The upper part of the box is the main idea implementation module. The locations of different modules are marked in different colors. 


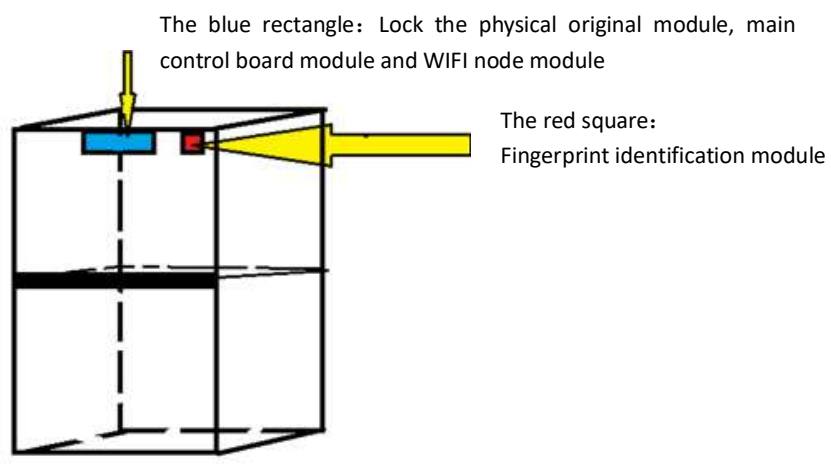

Figure 1 The Scheme Structure

The actual scheme architecture is shown in the figure below. Figure 2 is the actual architecture diagram. Figure 3 shows the fingerprint identification module from left to right, the electric locking module and the minimum system and the independent key.

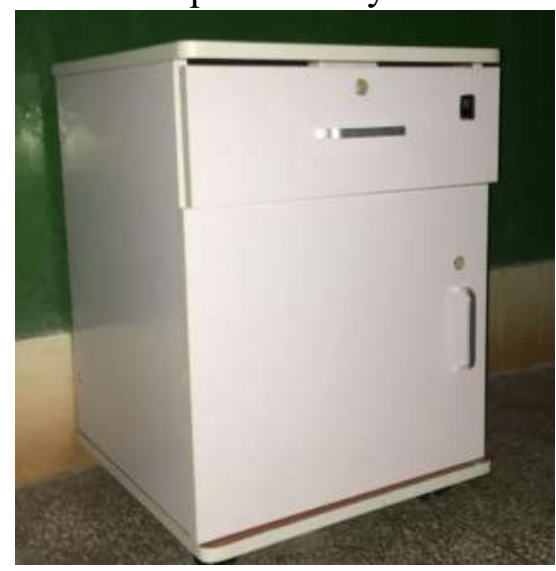

Figure 2 The Actual Diagram of the Façade

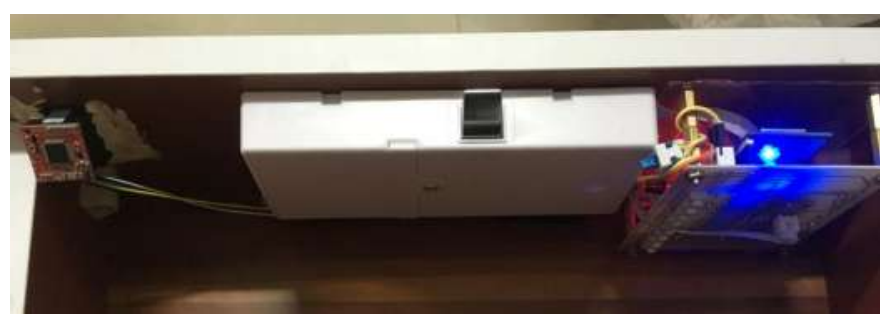

Figure 3 The Interior of the Actual Architecture Diagram

\section{Design and Construction of the Hardware Circuit}

\section{Selection of motor}

Plan 1: stepping motor.

For ordinary motor, the stepping motor can realize open loop control. It is to realize the Angle and speed control of the stepping motor through the number and frequency of the input of the input of the driver signal, and no feedback signal is needed. However, stepping motor is not suitable for use in the same direction for a long time. It is easy to burn the product, and the stepping motor cost is high [5].

Scheme 2: dc motor. Its transmission ratio is more detailed than classification, and has the advantages of wide selection and wide range of rotation speed. Range I $=2-28800$. It can precisely 
control the lock switch. Dc decelerator motor is compact, small in size, beautiful in shape and strong in overload. And it has low energy consumption and superior performance, and the speed reduction is up to 96 percent. It also has the advantages of small vibration and low noise. Its versatility is strong, so it is more convenient to maintain and the cost of maintenance is low. Especially in the production line, we can guarantee the normal production maintenance of the whole line by only a few transmission parts. It also adopts a new type of sealing device, so the protection performance is good and the environment is adaptable. It can work continuously in bad environment such as corrosion and humidity.

To sum up, we chose the scheme 2.

\section{Information Acquisition System}

The information acquisition system USES Arduino pcDuino fingerprint module and Wi-Fi module to collect password data. The data processing will be collected by STM32F103. After the data is processed, the dc motor is operated, and then the lock is turned on or off. Its schematic diagram is shown in figure 4.

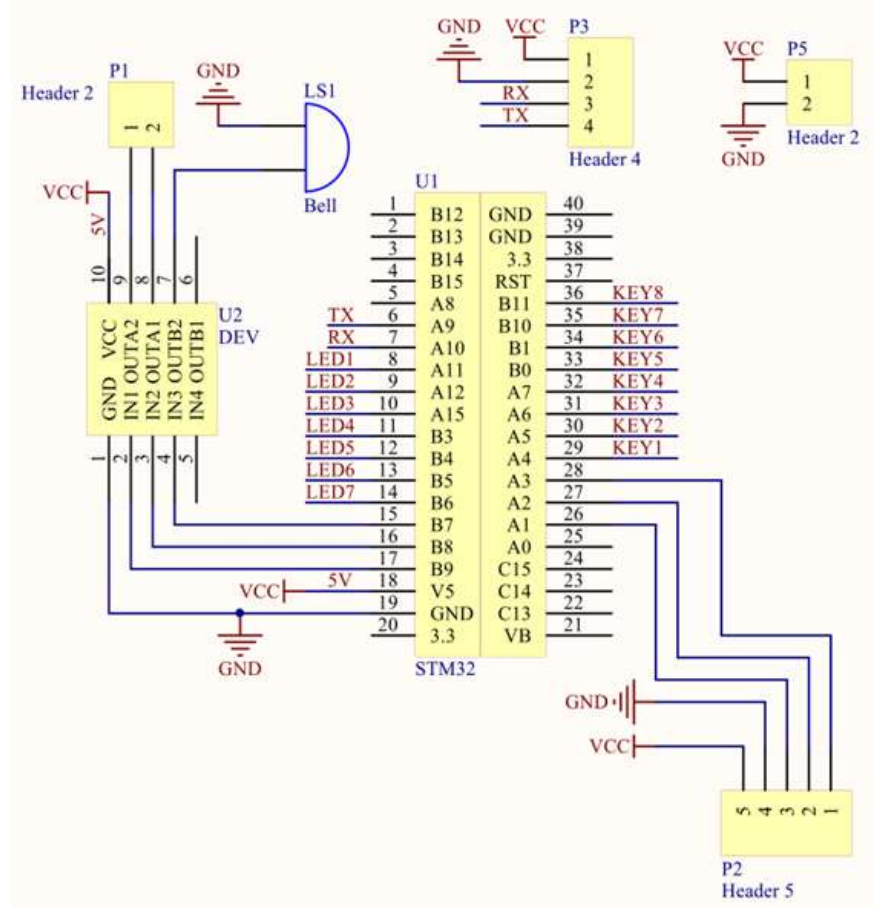

Figure 4 Schematic Diagram

The Fingerprint Version Control Module and Indicator Light Module

In Figure 5.KEY1 is to delete the current fingerprint.KEY2 is the input fingerprint.LED1 indicates the state of the lock switch. Indicates the color is red.LED2 indicates that the fingerprint function is deleted, indicating that the color is blue.LED3 indicates the status of the fingerprint function, indicating that the color is green. 

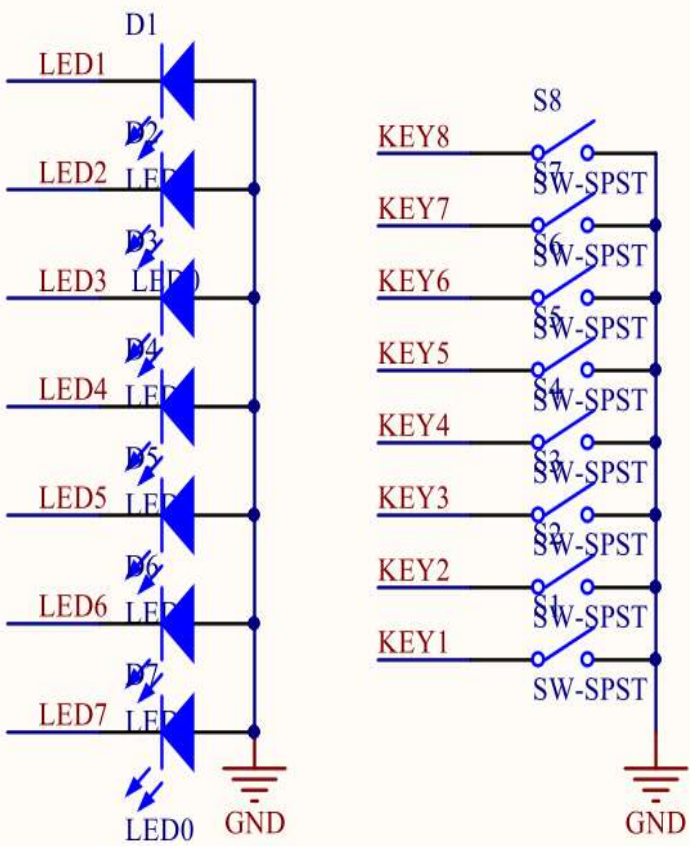

Figure 5 fingerprint version control and indicator light module

The Structure of the Hardware System

The data collection and processing part of the system structure diagram is shown in figure 6 :

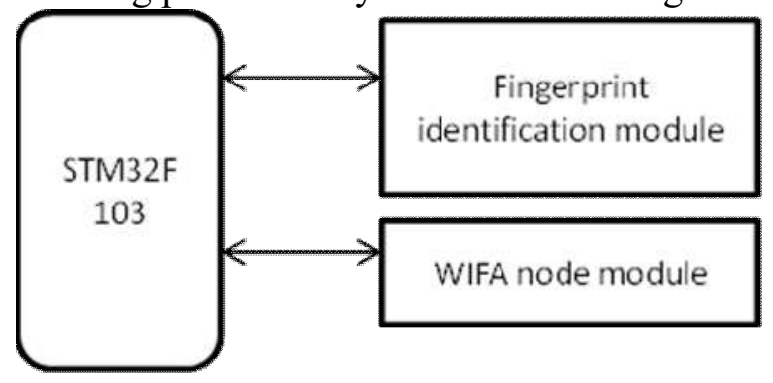

Figure 6 Data Collection and Processing Part of the System Structure Diagram

The structure diagram of the master control system is shown in Figure 7:

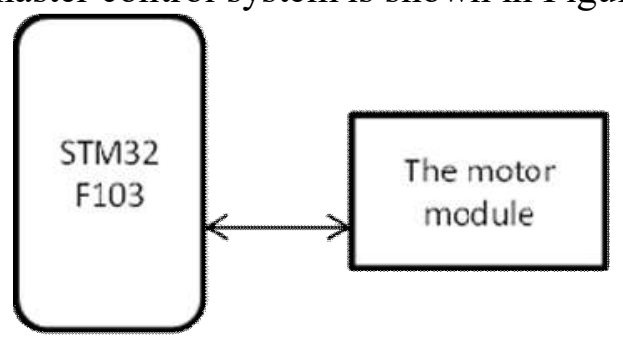

Figure 7 The Structure of the Main Control System

\section{The Oretical Analysis and Design}

There are two ways to "solve = lock": fingerprint unlocking, phone App to unlock. Fingerprint unlock as the main method of unlocking should be fast and accurate. If a particular situation occurs, the fingerprint identification is broken or damaged, and the mobile App unlock should be robust and responsive. 


\section{Conclusions}

This design adopts single chip microcontroller (STM32F103) as the minimum system. The independent keystroke and single chip are the control module of the fingerprint. Fingerprint identification module and WIFI module constitute information acquisition module. The dc motor and the lock - clasp physical components constitute the electric locking module. Three LED lights constitute a status indicator module. The syclstem is powered by a battery.

Working process of opening and locking:

Case 1: the fingerprint entry module collects the fingerprint information and then processes it through STM32F103, and then checks whether the input fingerprint matches the specified fingerprint. If the match is made through the rotation of the dc motor ( 5 seconds off the lock) and with a hint, if the input error is only returned to the prompt. The fingerprint unlocking will be banned more than 10 times in a row, and the alarm message will be sent to the mobile APP terminal, and the fingerprint opening will be banned after the alarm. The lock can only be used on the App side of the mobile phone, when the phone App is unlocked, the fingerprint identification will be removed.

Case 2: send the open lock information directly through the mobile App terminal to the WIFI reception module, and after the single-chip STM32F103 confirms the open lock information, the system will be unlocked ( 5 seconds off the lock) by rotating the dc motor.

Special case: when the battery is abnormal, it will be powered by the external power supply to ensure the normal operation of the system.

\section{References}

[1] Sarkar C, Uttama NSNA, Prasad R V, et al. DIAT: a scalable distributed architecture for IoT [J]. IEEE Internet of Things Journal, 2015,2(3): 230-239.

[2] Park D H , Bang H C, Pyo C S, et al. Semantic open IoT service platform technology[C] The 2014 IEEE World Forum on Internet of Things(WF-IoT), March 6-8, 2014, Seoul, Korea. New Jersey: IEEE Press, 2014:85-88.

[3] Cirani S, Picone M,Veltri L. IoT-Oas: an OAuth-based authorization service architecture for secure services in IoT scenarios[J]. IEEE Sensors Journal, 2015,15(2):1224-1234.

[4] Richardson L, Ruby S . RESTful Web Services[M]. Cambridge: O'Reilly Media, 2007: 199-204.

[5] Allamaraju S . RESTFUL Web Services Cookbook: Solutions for Improving Scalability and Simplicity[M]. Sunnyvale: Yahoo Press, 2010: 131-145. 